\title{
CATION COLLOIDAL PARTICLES IN ALKALINE-EARTH HALIDES
}

\author{
R. ALCALA and V. M. ORERA \\ Departamento de Física Fundamental. Universidad de Zaragoza, \\ Zaragoza, Spain
}

\begin{abstract}
Résumé. - Nous avons étudié la formation des colloïdes de calcium, strontium et barium par une forte irradiation électronique et par coloration additive des cristaux de $\mathrm{CaF}_{2}, \mathrm{SrF}_{2}$ et $\mathrm{BaF}_{2}$. Nous avons obtenu des renseignements détaillés sur la dépendance avec la température de l'efficacité de la formation des collö̈des par irradiation. La croissance des particules métalliques en échantillons colorés additivement et contenant des centres $\mathbf{F}$ et $\mathbf{M}$ a été étudiée pour différentes concentrations des centres colorés et températures de recuit.

En utilisant la théorie de Mie on a calculé les bandes d'absorption optique dues aux colloïdes métalliques. En considérant la pression qui fait la matrice sur les particules métalliques on a introduit diverses corrections aux constantes optiques des métaux. Les résultats expérimentaux obtenus sont en accord avec les calculs théoriques.

L'évolution des colloïdes a été étudiée selon différentes expériences de recuit. Un modèle de diffusion limitée a été utilisé pour rendre compte de la dépendance du rayon des colloïdes par rapport au temps de recuit.
\end{abstract}

\begin{abstract}
The formation of calcium, strontium and barium colloids both in heavily electron irradiated samples and in additively colored crystals of $\mathrm{CaF}_{2}, \mathrm{SrF}_{2}$ and $\mathrm{BaF}_{2}$ has been investigated. Detailed data on the temperature dependence of the efficiency of colloid formation by irradiation have been obtained. The growth of metallic particles in additively colored samples containing $\mathrm{F}$ and $\mathrm{M}$ centers has been studied for different color center concentrations and annealing temperatures.

The optical absorption bands due to metallic colloids have been calculated using the theory of Mie. To take account of the pressure exerted by the matrix on the metallic particles several corrections to the optical constants of the metals have been introduced. A good agreement between theoretical calculations and experimental results has been obtained.

The evolution of colloids along several thermal annealing experiments has also been investigated. A diffusion-limited model has been used which accounts for the dependence of the colloid radii with the annealing time.
\end{abstract}

1. Introduction. - It is well known that in alkali halides it is possible to form alkali metal colloids by thermal annealing of both additively colored [1] and heavily irradiated samples $[2,3]$.

The colloids produce an absorption band wich does not change its position and half-width when we cool the crystal down to $\mathrm{LN}_{2} \mathrm{~T}$ or $\mathrm{LHeT}$. On the other hand this band is very insensitive to optical bleaching and its position and width can be obtained using Mie's theory [4].

All these properties are characteristic of absorption bands due to colloids and we will use them to check that there are colloids in our crystals.

In the present work we report the formation of alkaline-earth metallic colloids in $\mathrm{CaF}_{2}, \mathrm{SrF}_{2}$ and $\mathrm{BaF}_{2}$ by heavy irradiation of samples at temperatures above room temperature and by thermal annealing of additively coloured crystals containing $F$ and $M$ centers.

The absorption bands due to colloids in these crystals appear in the visible region and they have the right properties that is, they do not bleach and do not change with the temperature of measurement.

To get an agreement between Mie's theory results and experimental data we must introduce several corrections to the optical properties of $\mathrm{Ca}, \mathrm{Sr}$ and $\mathrm{Ba}$ colloids in $\mathrm{CaF}_{2}, \mathrm{SrF}_{2}$ and $\mathrm{BaF}_{2}$ respectively. These corrections are due to the small size of colloids and to the pressure exerted by the matrix on the colloid.

We also report some experimental data concerning colloid's size evolution during several thermal annealing experiments.

It is possible to account for that evolution using a diffusion limited mechanism such as that of Lifshitz and Slyozov [5]. A similar treatment has been recently applied by Calleja and Agulló [6] to the nucleation and growth of Potassium colloids in $\mathrm{KCl}$ single crystals.

2. Experimental. - Single crystals of $\mathrm{CaF}_{2}, \mathrm{SrF}_{2}$ and $\mathrm{BaF}_{2}$ were purchased from Harshaw Chemical Co.

Some of them were irradiated in vaccuum at several temperatures using a Van de Graaf electron accelera- 


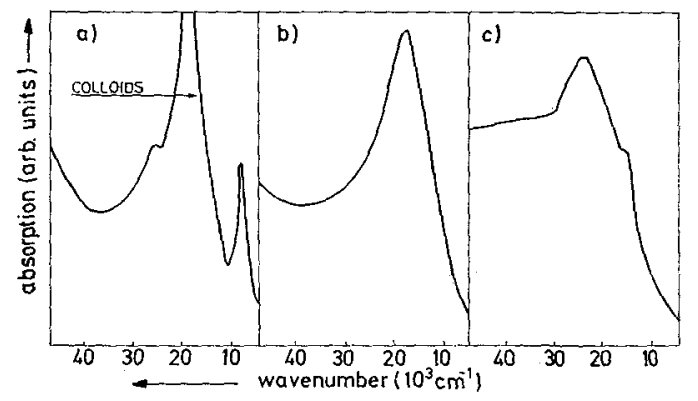

Fig. 1. - Optical absorption spectra corresponding to crystals of : a) $\mathrm{CaF}_{2}$; b) $\mathrm{SrF}_{2}$; c) $\mathrm{BaF}_{2}$, after heavy irradiation with $0.5 \mathrm{MeV}$ electron at $345 \mathrm{~K}, 488 \mathrm{~K}$ and $388 \mathrm{~K}$ respectively. The bands were measured at $\mathbf{R}$. T.

tor working at $0.5 \mathrm{MeV}$ and $1.5 \mu \mathrm{A} / \mathrm{cm}^{2}$. The samples were mounted in a thermostat with a variable temperature range from $80 \mathrm{~K}$ to $700 \mathrm{~K}$.

The additive coloration of the samples was performed following the method described by den Hartog [7].

The crystals were annealed in a furnace and cooled down again by putting them in contact with a copper plate.

The optical absorption was measured using a Cary 17 spectrophotometer.

3. Experimental results. - 3.1 IrRadiated SAMPLES. - We show in figure 1, the optical absorption spectra corresponding to crystals of $\mathrm{CaF}_{2}, \mathrm{SrF}_{2}$ and $\mathrm{BaF}_{2}$ after heavy irradiation with $0.5 \mathrm{MeV}$ electron at $72^{\circ} \mathrm{C}$, $215^{\circ} \mathrm{C}$ and $115^{\circ} \mathrm{C}$ respectively. The same spectra are obtained when we change the irradiation temperature, but the production efficiency is different. The efficiency as a function of irradiation temperature is shown in figure 2.

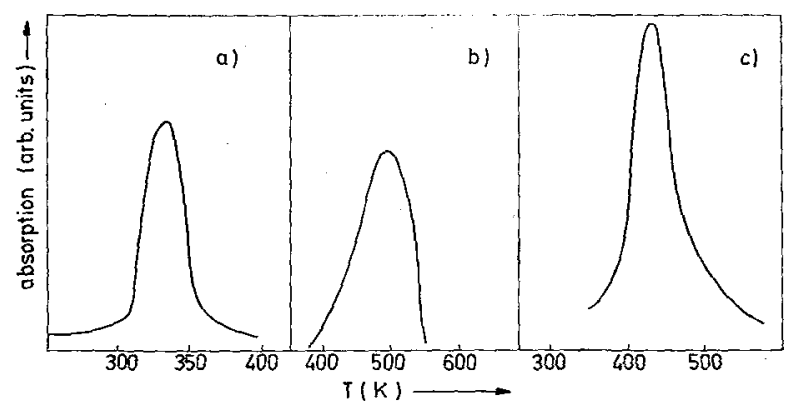

Fig. 2. - Temperature dependence of the efficiency of colloids production by $0.5 \mathrm{MeV}$ electron irradiation :a) $\left.\mathrm{CaF}_{2} ; b\right) \mathrm{SrF}_{2}$; c) $\mathrm{BaF}_{2}$.

The position and half-width of these bands do not change when we measure them at $\mathrm{LN}_{2} \mathrm{~T}$ or LHeT. They are also stable under optical bleaching.

From these properties we conclude that they can be due to colloids.
3.2 Additively COLORED SAMPLes. - Crystals of $\mathrm{Ca} \vec{F}_{2}, \mathrm{SrF}_{2}$ and $\mathrm{BaF}_{2}$ containing $\mathrm{F}$ and $\mathrm{M}$ centers were annealed at several temperatures and times.

In figure 3, we show the absorption spectra of one sample of each material, before and after an annealing experiment. We can see that the colloid bands correspond to those obtained by irradiation and that have the same properties with respect to optical bleaching and temperature of measurement.

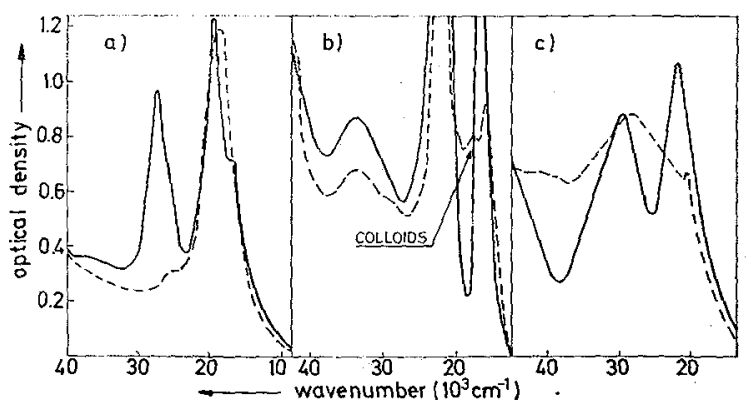

Frg. 3. - Absorption spectra of : a) $\left.\left.\mathrm{CaF}_{2} ; b\right) \mathrm{SrF}_{2} ; c\right) \mathrm{BaF}_{2}$, aditively colored, - before and -.- after an annealing experiment (see the text).

The spectrum corresponding to $\mathrm{SrF}_{2}$ is very rich. It has several impurity bands which mask the colloid band. Anyway we can identify that band because its properties coincide approximately with that of the band obtained in irradiated samples.

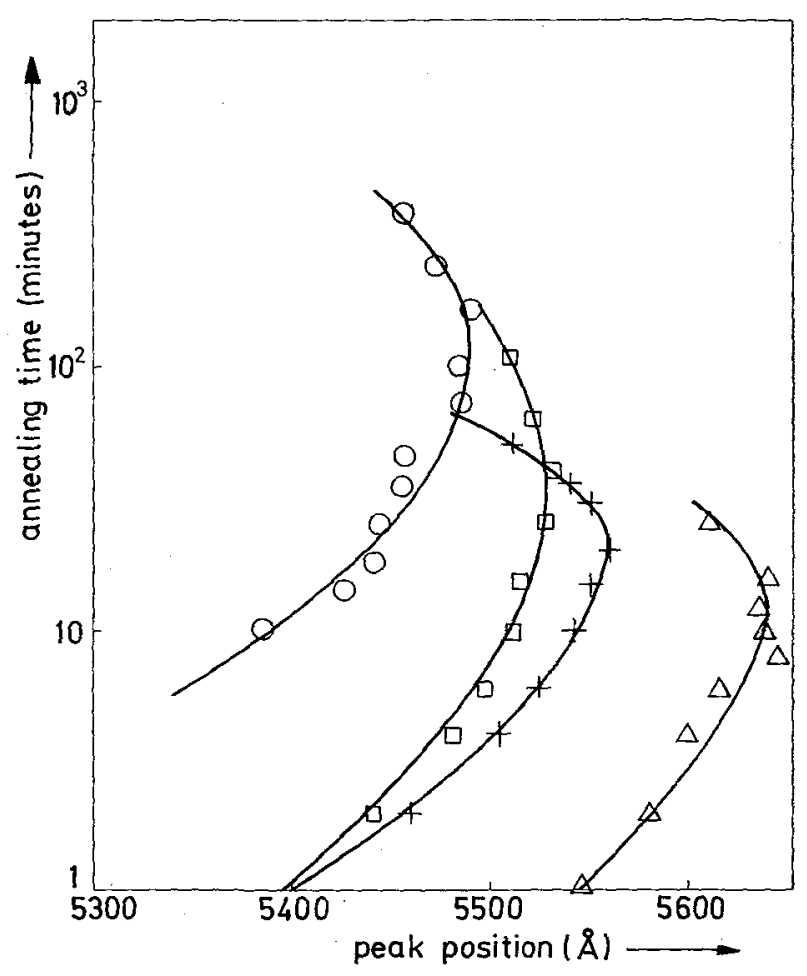

Fig. 4. - Peak positions of $\mathrm{Ca}$ colloids band in $\mathrm{CaF}_{2}$ for different annealing times and the following temperatures $\triangle: 548 \mathrm{~K}$, $\times: 518 \mathrm{~K}, \square: 511 \mathrm{~K}, \mathrm{O}: 483 \mathrm{~K}$ 
The $F$ and $M$ centers initial concentrations do not influence the final band position, so we will use only the data corresponding to an initial concentration of $2 \times 10^{17} \mathrm{~F}$ centers.

The final position of the bands depends on annealing time and temperature.

In figure 4 we show the evolution of colloid band positions vs. annealing time for $\mathrm{CaF}_{2}$, at different temperatures. We do not include data for $\mathrm{SrF}_{2}$ and $\mathrm{BaF}_{2}$ because the overlapping with some impurity bands masks small changes in position.

As we can see, first the colloid band shifts toward longer wavelength and after it goes back to lower wavelength. This second stage corresponds to a decrease in the coloration of samples until finally the coloration disappears.

4. Theoretical calculations of colloids bands. The absorption bands produced by metallic colloids can be calculated theoretically using Mie's theory [4].

According to Mie's results the absorption coefficient « $\gamma$ » corresponding to $N$ particles of volume $V$ is given by

$\gamma=N \frac{\lambda^{2}}{2 \pi} \mathrm{R}\left\{\sum_{l=1}^{\infty}(-i)^{l+1} l(l+1)\left({ }^{e} B_{l}+{ }^{m} B_{l}\right)\right\}$

where $\lambda$ is the wavelength of light in the outher medium and ${ }^{e} B_{1}$ and ${ }^{m} B_{1}$ are coefficients that depend on the wavelength of light, the size of colloids and the dielectric constants of outer medium and metallic colloids. $\mathrm{R}$ means real part.

Before putting in (1) the values of colloid's dielectric constants we must introduce several corrections.

The dielectric constant of a metal can be written as :

$$
\widehat{\varepsilon}=\varepsilon_{1}+i \varepsilon_{2}
$$

where

$$
\widehat{\varepsilon}_{1}=\varepsilon_{0}-\frac{\omega_{p}^{2}}{\omega^{2}+\omega_{0}^{2}}+B_{1}(\omega)
$$

and

$$
\varepsilon_{2}=\frac{\omega_{\mathrm{p}}^{2} \omega_{0}}{\omega\left(\omega^{2}+\omega_{0}^{2}\right)}+B_{2}(\omega) .
$$

Here: $\varepsilon_{0}=1+4 \pi N_{\mathrm{X}} \alpha_{\mathrm{I}}$ where $N_{\mathrm{I}}$ is the atomic concentration and $\alpha_{1}$ the atomic polarizability :

$$
\omega_{\mathrm{p}}^{2} \equiv \frac{4 \pi n e^{2}}{m_{\mathrm{opt}}},
$$

$m_{\text {opt }}$ being the optical electron mass. As we do not know the optical mass we will take instead the thermal effective mass which is usualy close to the optical effective mass, $n$ is the electron concentration ;

$$
\omega_{0} \equiv \frac{1}{\tau_{0}}
$$

where $\tau_{0}$ is the relaxation time for electron conductivity and $B_{1}(\omega)$ and $B_{2}(\omega)$ are the interband contributions.

The first correction is a change in $\omega_{0}$ due to the small size of colloids. Following Kreibig [8] we have for the size dependence of $\omega_{0}$

$$
\omega_{0}(R)=\omega_{0}+\frac{v_{\mathrm{F}}}{R}
$$

where $\omega_{0}$ is the uncorrected value, $v_{\mathrm{F}}$ is the Fermi velocity of electrons and $R$ is the particle radius.

The other correction is a change in $\omega_{\mathrm{p}}$ due to the pressure exerted by the matrix on the colloid.

We have not taken in account the changes of $B_{1}(\omega)$ and $B_{2}(\omega)$ with that pressure but we have checked that they are small for our pressure range by using the theory given by Ashcrofft and Sturm [9] for the interband contribution to the dielectric constant, taking for the pseudopotential the model given by Cohen and Heine [10].

To find the change in $\omega_{\mathrm{p}}$ we first calculate the pressure on the colloid.

If we assume that there is complete coherency between the lattice of colloids and the cation lattice in the matrix as it has been shown for $\mathrm{Ca}$ colloids in $\mathrm{CaF}_{2}$ by Murr [11], we find out that due to the difference in lattice parameter between the alkaline-earth halide and the metal, the colloid is under pressure.

We can calculate that pressure, following elasticity theory, if we take the colloid as a spherical metallic particle within a cavity in the crystal that, due to the different lattice parameter, has a smaller radius than the colloid. The resultant pressure is :

$$
P=4 \mu \frac{3 B_{\mathrm{p}} a^{*}}{3 B_{\mathrm{p}}+4 \mu}
$$

where :

$\mu$ is the Lamé coefficient of the matrix $B_{\mathrm{p}}$ the metal bulk modulus and

$a^{*}=\frac{v_{\mathrm{m}}-v_{\mathrm{h}}}{v_{\mathrm{h}}}$ being $v_{\mathrm{m}}$ and $v_{\mathrm{h}}$ the molar volumes of metal and alkaline-earth halide respectively.

The pressure values obtained are $7.8 \mathrm{kbar}$ for $\mathrm{Ca}$, $13.8 \mathrm{kbar}$ for $\mathrm{Sr}$ and $4.5 \mathrm{kbar}$ for $\mathrm{Ba}$ which will produce a change in the volume of the metal such that if $v_{0}$ is the volume without pressure and $v_{\mathrm{p}}$ the volume under pressure $; v_{p} / v_{0}$ takes the values $0.951,0.890$ and 0.959 for $\mathrm{Ca}, \mathrm{Sr}$ and $\mathrm{Ba}$ respectively.

To see the influence of this volume change on $\omega_{p}$ we have used the following procedure.

The conductivity $\sigma$ can be written as :

$$
\sigma=\frac{\omega_{\mathrm{p}}^{2} \tau\left(k_{\mathrm{F}}\right)}{4 \pi}
$$

where $\tau\left(k_{F}\right)$ is the relaxation time of conduction electrons at the Fermi level.

Now the change in $\sigma$ with the pressure can be calculated using the data given by Mott [12]. 
The change of $\tau\left(k_{\mathrm{F}}\right)$ with the pressure can be obtained using a free electron model [13] and some of the results given in [14].

In this way we have obtained (V. M. Orera and R. Alcalá unpublished) for $\omega_{\mathrm{p}}(0) / \omega_{\mathrm{p}}(\mathrm{p})$ the values 1.11 for $\mathrm{Ca}, 1.65$ for $\mathrm{Sr}$ and 1.02 for $\mathrm{Ba}$ where $\omega_{\mathrm{p}}(0)$ is the plasma frequency without pressure and $\omega_{\mathrm{p}}(\mathrm{p})$ the corrected value.

Using the new values for $\omega_{0}$ and $\omega_{p}$ we can calculate the dielectric constants $\varepsilon_{1}$ and $\varepsilon_{2}$ for $\mathrm{Ca}, \mathrm{Sr}$ and $\mathrm{Ba}$ colloids and apply Mie's theory. We show in figure 5 the position of colloid bands in $\mathrm{CaF}_{2}, \mathrm{SrF}_{2}$ and $\mathrm{BaF}_{2}$, corresponding to several colloid sizes.
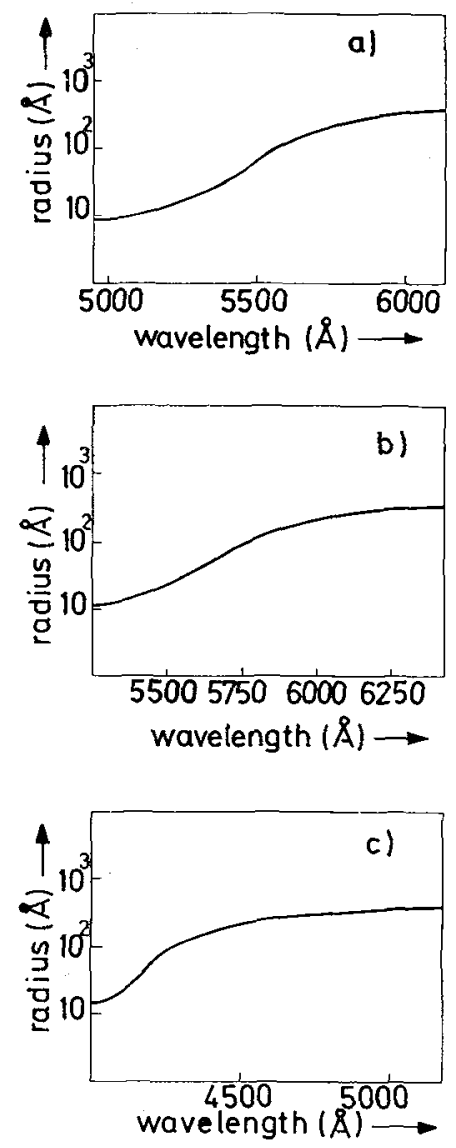

FIG. 5. - a) Theoretical values of the position of Ca colloids bands in $\mathrm{CaF}_{2}$ versus colloid radii ; $b$ ) The same for $\mathrm{SrF}_{2}$ and c) for $\mathrm{BaF}_{2}$.

We can now compare our experimental and theoretical results. To do that we calculate using figure 5 , the colloid radius that correspond to the peak position we measure. From that radius we can now calculate the absorption band using Mie's theory.

We report in figure 6 the theoretical band calculated in that way, together with several points of the experimental bands obtained in our electron irradiation experiments.

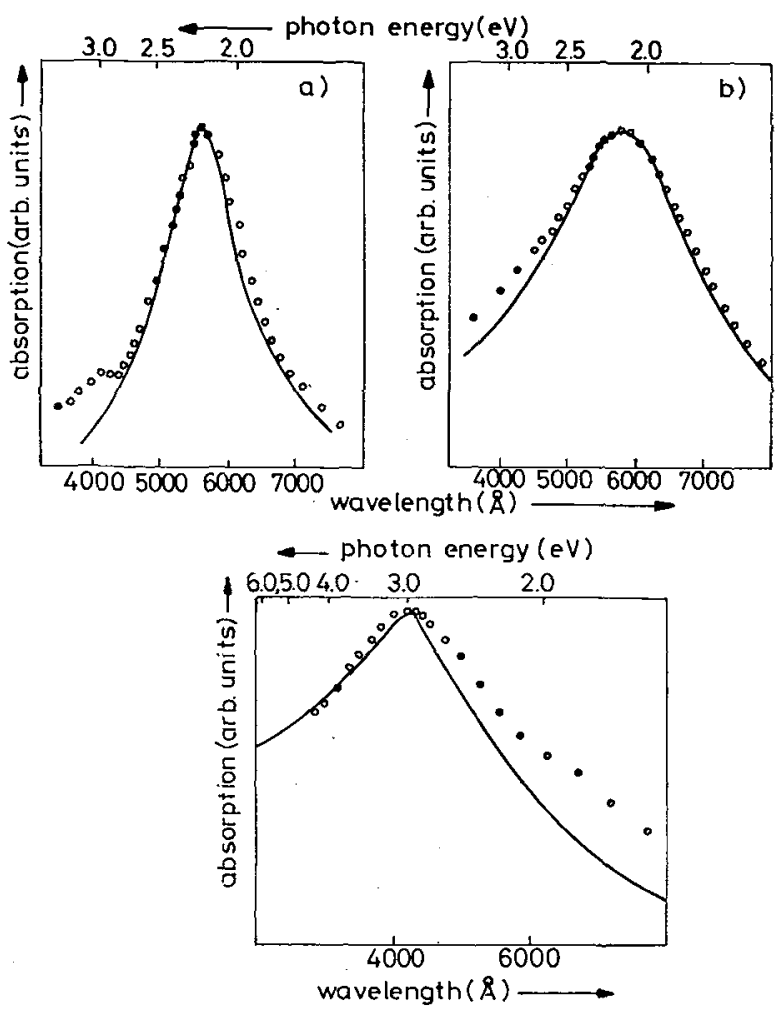

Fig. 6. - a) Comparison between theoretical and experimental (by points) absorption bands of $\mathrm{Ca}$ colloids in $\mathrm{CaF}_{2} 0.5 \mathrm{MeV}$ electron irradiated at $345 \mathrm{~K} ; b$ ) The same for $\mathrm{SrF}_{2}$ irradiated at $488 \mathrm{~K}, c)$ The same for $\mathrm{BaF}_{2}$ irradiated at 388 .

The same agreement is obtained in $\mathrm{CaF}_{2}$ and $\mathrm{BaF}_{2}$ for additively colored samples.

5. Size evolution of colloids using annealing experiments. - Using the results reported in figure 5 we can now calculate the colloid radii that correspond to the different peak positions we have drawn in figure 4 .

In that way we can follow the evolution of colloid radius with annealing time and temperature. As we have said before we will work only with $\mathrm{CaF}_{2}$.

We shown in figure 7 the colloid radius as a function of time, for several temperatures, during the first stage that is, before the coloration begins to fall down.

We represent $R^{3}$ vs.t, being $R$ the colloid radius and $t$ the annealing time.

We get a straight line for each annealing temperature with an increasing slope when we raise the temperature.

It is possible to give an explanation for that kind of evolution using a mechanism such as that proposed by Lifshitz and Slyozov for the kinetics of precipitation from a supersaturated solid solution [5].

A modification of a similar mechanism proposed by Chakraverty [15] has been applied recently by Calleja and Agullo to the nucleation and growth of Potassium colloids in $\mathrm{KCl}$ [6]. 


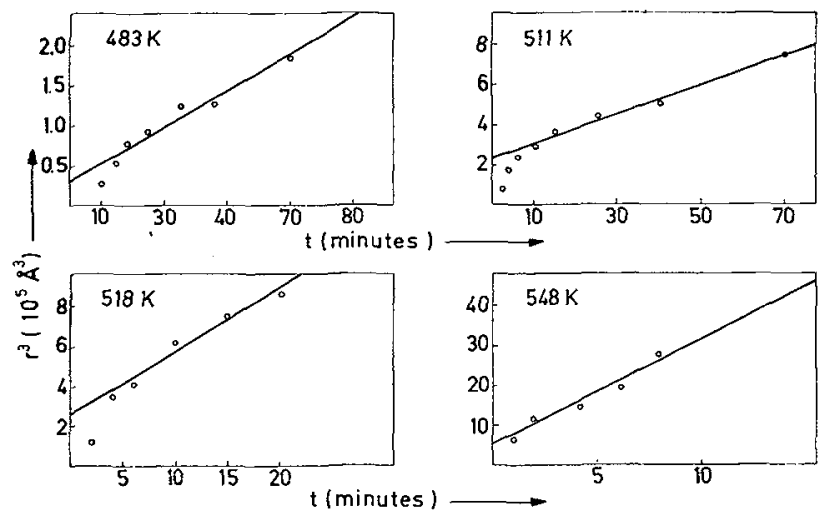

FIG. 7. - Plot of $R^{3} v s_{0} t$ for Ca colloids in $\mathrm{CaF}_{2}$ at different temperatures.

Following the treatment given in [15] we find a distribution function that gives the number of particles with radius between $R$ and $R+\mathrm{d} R$ at a time $t$.

That function is given by :

$$
f(\rho, t)=\psi(\rho) . \Phi(t)
$$

where

$$
\begin{array}{ll}
\psi(\rho)=\frac{3 \rho^{2} \exp \left(\frac{3}{2 \rho-3}\right)}{\left(\frac{3}{2}-\rho\right)^{11 / 3}(\rho+3)^{7 / 3}} ; \rho<\frac{3}{2} \\
\psi(\rho)=0 \quad ; \rho<\frac{3}{2}
\end{array}
$$

and $\Phi(t)$ is only function of time where $\rho=R / R_{\mathrm{c}}$, being $R_{\mathrm{c}}$ a parameter, function of time, called critical radius and such that all the particles with radius bigger than $R_{\mathrm{c}}(t)$ grow and all the particles with radius smaller than $R_{\mathrm{c}}(t)$ disolve and disappear.

When we try to calculate the colloid absorption band using Mie's theory we find that the absorption coefficient $\gamma$ is proportional to $N \times V$, where $N$ is the number of particles and $V$ the volume of each particle. So we are not interested in $\psi(\rho)$ but in $\psi(\rho) \times R^{3}$ or $\psi(\rho) \times \rho^{3} \times R_{\mathrm{c}}^{3}$.

This function has been drawn in figure 8 for an arbitrary value of $R_{\mathrm{c}}$. As the function is very sharp we can use the $\rho$ value that correspond to the maximum to calculate the colloid band. That value is $\rho_{\mathrm{m}}=1.189$.

Following with Lifshitz's treatment the evolution of colloid radius that correspond to $\rho_{\mathrm{m}}$ is given by

$$
R(t, T)=\rho_{\mathrm{m}}\left(\bar{R}_{1}^{3}+\left(\frac{4}{9} D \alpha t\right)\right)^{1 / 3}
$$

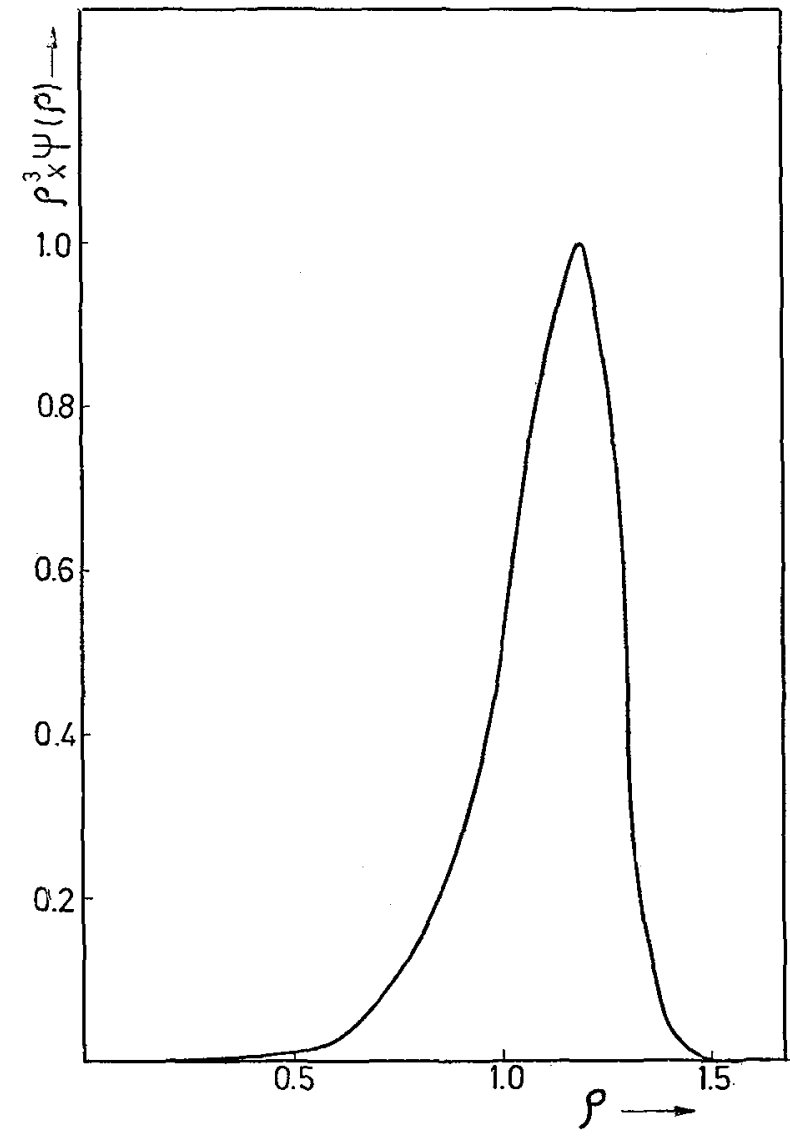

FIG. 8. - Distribution function $\psi(\rho) . \rho^{3}$ giving the volume distribution of colloids for an arbitrary $R_{\mathrm{c}}$. The maximum corresponds to $\rho_{\mathrm{m}}=1.19$.

where $\bar{R}_{1}^{3}$ is an initial dimension, $D$ is the diffusion coefficient of solute particles, and $\alpha=\frac{2 \sigma}{k T} v C_{\infty}$ being $\sigma$ the interface surface tension, $V$ the volume of solute particles and $C_{\infty}$ the equilibrium concentration of solute.

That is the kind of evolution we have found in our experimental measurements of colloid radii, as we see in figure 7.

So, we can conclude that there is agreement between theory and experiments if we apply to the size evolution of colloids the bulk diffusion limited mechanism proposed by Lifshitz and Slyozov.

Acknowledgments. - We are thankful to Dr. A. E. Hughes for his criticism and suggestions to a part of this paper and to the A.E. R. E. for the irradiation facilities. 


\section{References}

[1] Schulman, J. H. and Compron, W. D., Color Centers in Solids (Pergamon Press, London) 1962.

[2] Compton, W. D., Phys. Rev. 107 (1957) 1271.

[3] Pappu, S. V. and MCCarthy, K. A., J. Phys. \& Chem. Solids 32 (1971) 1287.

[4] MiE, G., Ann. Phys. 25 (1908) 377.

[5] Lifshitz, I. M. and Slyozov, J. Phys. \& Chem. Solids 19 (1961) 35.

[6] Calleja, J. M. and Aqulló-López, F., Phys. Status Solidi (a) 25 (1974) 473.

[7] Den Hartog, H. W., Thesis, University of Groningen (1969).
[8] Kreibig, U. and Fragstein, C. V., Z. Phys. 224 (1969) 307.

[9] Ashcrofft, N. W. and Sturm, K., Phys. Rev, B 3 (1971) 1898.

[10] Cohen, M. L. and Heine, V., Sol. Stat. Phys. 24 (1970) 55.

[11] Murr, L. E., Phys. Status Solidi (a) 22 (1974) 239.

[12] Motr, N. F., Proc. Phys. Soc. (London) 46 (1934) 680.

[13] SeITz, F., The Modern Theory of Solids (McGraw-Hill, New York) 1940.

[14] Lawson, A. W., Prog. Met. Phys. 6 (1956) 1.

[15] Chakraverty, B. R., J. Phys. \& Chem. Solids 28 (1967) 2401. 